The effect of mandi bul ar set back or two-j aws surgery on pharyngeal ai rway among di ffer ent gender $\mathrm{s}$

\begin{tabular}{|l|l|}
\hline 著者 & $\begin{array}{l}\text { Kagan Deger I i yurt, Ueki Koi chi r o, Hashi ba } \\
\text { Yukar i, Nar ukawa Kohei, Si nsek Bar i s, Okabe } \\
\text { Kat suhi ko, Nakagawa Ki yomasa, Yamantt o } \\
\text { Et suhi de }\end{array}$ \\
\hline $\begin{array}{l}\text { j our nal or } \\
\text { publ i cat i on t i t I e }\end{array}$ & $\begin{array}{l}\text { I nt er nat i onal J our nal of Oral and } \\
\text { Naxi I I of aci al Sur gery }\end{array}$ \\
\hline vol une & 38 \\
\hline number & 6 \\
\hline page r ange & $647-652$ \\
\hline year & $2009-06-01$ \\
\hline URL & ht t p: //hdl . handl e. net /2297/17429 \\
\hline
\end{tabular}




\section{THE EFFECT OF MANDIBULAR SETBACK OR TWO-JAWS SURGERY ON PHARYNGEAL AIRWAY AMONG DIFFERENT GENDERS}

KAGAN DEGERLIYURT, DDS ${ }^{2}$ KOICHIRO UEKI, DDS, PhD ${ }^{1}$ YUKARI HASHIBA, DDS, PhD ${ }^{1}$ KOHEI MARUKAWA, DDS, PhD ${ }^{1}$ BARIS SIMSEK, DDS, PhD ${ }^{2}$ KATSUHIKO OKABE, DDS ${ }^{1}$ KIYOMASA NAKAGAWA, DDS, PhD ${ }^{1}$ and ETSUHIDE YAMAMOTO, DDS, $\mathrm{PhD}^{1}$

1. Department of Oral and Maxillofacial Surgery, Graduate School of Medicine, Kanazawa University, 13-1 Takaramachi, Kanazawa 920-8641, Japan

2. Gazi University, School of Dentistry, Department of Oral and Maxillofacial Surgery, 8. Cad 82. Sok. 06510 Emek, Ankara, TURKEY

Address Correspondence To: Kagan Degerliyurt, DDS

Gazi University, School of Dentistry, Department of Oral and Maxillofacial Surgery,

8. Cadde 82. sok. 06510 Emek, Ankara, TURKEY

Tel: +903122034339 Fax: +90312223 9226

Email: mkdegerliyurt@gazi.edu.tr

Keywords: Mandibular setback, Bimaxillary surgery, Pharyngeal airway, Gender, Computed Tomography

Short title: THE EFFECT OF MANDIBULAR SETBACK OR TWO-JAWS SURGERY ON PHARYNGEAL AIRWAY AMONG DIFFERENT GENDERS 
THE EFFECT OF MANDIBULAR SETBACK OR TWO-JAWS SURGERY ON PHARYNGEAL AIRWAY AMONG DIFFERENT GENDERS 


\section{THE EFFECT OF MANDIBULAR SETBACK OR TWO-JAWS SURGERY ON PHARYNGEAL AIRWAY AMONG DIFFERENT GENDERS}

\section{Abstract}

Cephalometric studies have revealed that there were significant sexual differences in the size of the pharyngeal airway space. The purpose of this study was investigate and compare the morphologic changes after mandibular setback or two jaws surgery on pharyngeal airway between females and males with computed tomography. The sample is consisted of 34 female and 13 male patients in 4 groups who had been diagnosed with Class III skeletal deformities and had been treated by mandibular setback or bimaxillary surgery (maxillary advancement and mandibular setback). Anteroposterior, lateral, cross sectional area dimensions of the airway at the level of soft palate and base of tongue was measured pre- and postoperatively on computed tomography images. In mandibular setback group, anteroposterior and cross sectional area of the pharyngeal airway at the level of both soft palate and base of tongue were significantly reduced for either females or males. $(P<.05)$ In two-jaws surgery group, only midsagittal anteroposterior dimensions of the pharyngeal airway at the level of soft palate and base of tongue were significantly decreased for either females or males. $(P<.05)$ Mann Whitney $U$ test revealed that the difference between any values measured between males and females who received either BSSO setback surgery or two-jaws surgery for the treatment of class III anteroposterior discrepancy were statistically insignificant. $(P>.05)$ This study suggested that oropharyngeal airway measurements which are most important to the patency of the airway does not demonstrate sex dimorphism. 


\section{THE EFFECT OF MANDIBULAR SETBACK OR TWO-JAWS SURGERY ON PHARYNGEAL AIRWAY AMONG DIFFERENT GENDERS}

\section{Introduction}

Muscles in the pharyngeal region do not act independently, but rather work together to achieve equilibrium; otherwise, the patency of the pharyngeal airway would be jeopardized ${ }^{13}$. It has been shown that the respiratory related activity of the muscles is significantly different between males and females ${ }^{15}$. Additionaly, cephalometric studies have revealed that there were significant sexual differences in the size of the oropharynx. These data suggest that gender may affect the morphology of the pharyngeal airway following surgical correction of mandibular prognathism ${ }^{13,19,21}$.

Mandibular setback osteotomy has routinely been used as an orthognathic surgical procedure for mandibular prognathism ${ }^{9}$. Recent studies indicated that isolated mandibular anteroposterior excess occurs in only approximately $20 \%$ to $25 \%$ of mandibular prognathism cases $^{16}$. Advances in knowledge and techniques led the corrective surgery to progress mainly towards two-jaws surgical procedures ${ }^{2}$. Recently, mandibular setback surgery decreased in frequency to fewer than $10 \%$ of mandibular prognathism patients, whereas two-jaws surgery was preferred in about $40 \%$ of patients. Maxillary advancement alone is performed in the remaining patients ${ }^{1}$.

Either mandibular setback surgery or two jaws surgery can improve occlusion, masticatory function and esthetics by markedly changing the position of the mandible and maxilla. Studies have also shown changes in the position of the tongue and hyoid bone resulting in narrowing of the pharyngeal airway space $\mathrm{e}^{2,4,6-9,23}$. PAS narrowing has been implicated in the development of obstructive sleep apnea (OSA) $)^{5,17,22}$.

The purpose of this study was investigate and compare the morphologic changes after mandibular setback or two jaws surgery on pharyngeal airway between females and males 
with computed tomography. We examined several values which obtained in two different part of the pharyngeal airway with special attention to sexual dimorphism.

\section{Patients and methods}

This is a retrospective study of 47 japanese patients ( 34 females, 13males) who were treated with either a mandibular surgery or a combined maxillary and mandibular surgery for the correction of Class III anteroposterior discrepancy. The average age of the patients was 23.3 \pm 6.3 years old, with a range of 16 to 42 .

All 47 patients had pre- and postoperative orthodontic treatment. The surgical procedure in all cases consisted of either bilateral sagittal split ramus osteotomy (BSSO) or Le Fort I osteotomy combined with BSSO. The osteotomy sites were fixed rigidly with either titanium or polylactic/polyglycolic acid miniplates. They were divided into four groups based on the gender and type of orthognathic surgery they had received. Group A is consisted of 17 female patients (25.6 \pm 7.6 years old, ranging from 17 to 42$)$ while group B is consisted of 7 male patients (17.8 \pm 2.5 years old, ranging from 17 to 25$)$ who underwent BSSO setback with rigid fixation. Group C is consisted of 17 female patients ( $22.1 \pm 6.1$ years old, ranging from 16 to 38) while Group D is consisted of 6 male patients $22.4 \pm 8.2$ years old ranging from 20 to 31 ) who underwent BSSO setback and Le Fort I maxillary advancement with rigid fixation. The mean amount of setback in group A was $6.6 \pm 3.5 \mathrm{~mm}$ for the right side and $7.5 \pm 3.3 \mathrm{~mm}$ for the left side, ranging from $2 \mathrm{~mm}$ to $13 \mathrm{~mm}$ for both sides, while the mean amount of setback was $7.2 \pm 1.8 \mathrm{~mm}$ for the right side, ranging from $4 \mathrm{~mm}$ to $9 \mathrm{~mm}$, and $5.6 \pm 3.7 \mathrm{~mm}$ for the left side, ranging from $3 \mathrm{~mm}$ to $11 \mathrm{~mm}$, for Group B. In group C, the mean amount of setback was $7.1 \pm 2.8 \mathrm{~mm}$ for the right side, ranging from $3 \mathrm{~mm}$ to $13 \mathrm{~mm}$, and $6.6 \pm 3.1 \mathrm{~mm}$ for the left side, ranging from $1 \mathrm{~mm}$ to $12 \mathrm{~mm}$, while the mean amount of setback was $6.7 \pm 3.0 \mathrm{~mm}$ for the right side, ranging from $4 \mathrm{~mm}$ to $12 \mathrm{~mm}$, and $6.2 \pm 3.1 \mathrm{~mm}$ for the left side, ranging from $1 \mathrm{~mm}$ to $12 \mathrm{~mm}$, for Group D. All the patients had maxillomandibular fixation (MMF) 
for approximately 1 week postoperatively. Guiding elastics were placed after release from MMF.

The mean body mass index (BMI) and standard deviation of BMI for the patients were

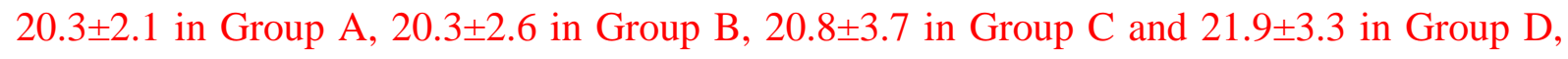
respectively. Mann Whitney U's test revealed no significant difference between either group $\mathrm{A}$ and $\mathrm{B}(\mathrm{P}>$.9999) or group $\mathrm{C}$ and $\mathrm{D}(\mathrm{P}=0.6241)$.

The CT was performed within a week before the surgery and postoperative CT evaluation was performed at least 3 months postoperatively for all patients as referred by many authors ${ }^{2,7-9}$. Informed consents were obtained from all patients and the study was approved by Kanazawa University Hospital Committee on Human Research.

The CT was performed while the patients were placed in the gantry with the tragocanthal line perpendicular to the ground for CT scanning. The patients were instructed to remain still and to avoid swallowing during the scanning process. The CT scans were obtained at the same radiology department by skilled radiology technicians using a high speed advantage type CT generator ( Light Speed Plus: GE healthcare, Milwaukee, WI, USA) with each sequence taken $1.25 \mathrm{~mm}$ apart for 3D reconstruction image ( $120 \mathrm{kV}$, average $150 \mathrm{~mA}, 0.7$ sec/rotation, helical pitch 0.75). The resulting images were stored in the attached workstation computer (Advantage workstation ver. 4.2: GE healthcare, Milwaukee, WI, USA) and 3D reconstruction was performed using volume rendering method. The ExaVision LITE version 1.10 medical imaging software (Ziosoft, Inc, Tokyo, Japan) was used for 3D morphologic measurements.

The exclusion criteria was set as previous orthognathic surgery, obesity, craniofacial anomaly (Cleft lip, palate, alveolus) and OSA.

The pre- and postoperative upper airway of each patient was studied in two levels: (Fig.1) 
1. The level of most superior anterior point of second cervical spine (C2) parallel to SellaNasion line to evaluate the airway between soft palate (SP) and posterior pharyngeal wall (PPW) or between lateral pharyngeal walls (LPWs)

2. The level of most superior anterior point of third cervical spine (C3) parallel to SellaNasion to evaluate the airway between base of the tongue (BoT) and PPW or between LPWs. A set of three values was obtained at each airway level: (1) Anteroposterior (AP) dimension on the midsagittal plane (Fig. 2); (2) maximum lateral dimension (LAT) in an orientation perpendicular to the midsagittal plane (Fig. 2); (3) cross sectional area of the airway (CSA) (Fig. 3). The measurement of the CSA was performed simply by following the perimeter of the airway with the cursor. No tracing or digitizing of the axial images was required because the software available automatically calculated the area contained within the scribbled line.

All statistical analyses were carried out using StatView ${ }^{\mathrm{TM}}$ version 4.5 software (ABACUS Concepts, Inc, Berkeley, CA, USA). The arithmetic mean and standard deviation were calculated for each variable. Wilcoxon signed rank test, with statistical significance being inferred at $P<.05$, was used to evaluate the differences between preoperative and postoperative pharyngeal airway morphology in each group. Differences between the gender groups who received the surgical procedure were analysed using Mann Whitney $U$ test and $P<.05$ was considered significant. Mann Whitney $\mathrm{U}$ test was used because of unequal sample size.

All CT images were evaluated and AP, LAT and CSA dimensions were measured by an author (K.D). Fifteen patients were selected randomly and CT images were measured again 10 days later. Wilcoxon signed rank test was applied to the first and second measurements. The difference between first and second measurements of 15 CT images was statistically insignificant ( $>$ >.05). 


\section{Results}

BSSO setback surgery group.

Wilcoxon signed rank test results comparing preoperative and postoperative linear and area measurements of the pharyngeal airway and percentage of difference between preoperative and postoperative values for group A and B are shown in Table I. 4 of 6 values in both studied levels were significantly reduced for either group A or group B. $(P<.05)$

Mann Whitney U test revealed that the difference between any values measured for group A and group B who received BSSO for the treatment of class III antreroposterior discrepancy were statistically insignificant. $(P>.05)$ The results of Mann Whitney U test for group A and B are shown in Table II.

Two-Jaws surgery group

Wilcoxon signed rank test results comparing preoperative and postoperative linear and area measurements of the pharyngeal airway and percentage of difference between preoperative and postoperative values for group C and D are shown in Table III. In both groups, only midsagittal AP dimensions of the pharyngeal airway at the level of SP and BoT were significantly decreased. $(P<.05)$ Although AP, LPWs and CSA dimensions of the pharyngeal airway decreased in both groups, there were no statistically significant reduction in LPWs and CSA dimensions between pre- and post-operative measurements. $(P>.05)$

Mann Whitney $\mathrm{U}$ test revealed that the difference between any values measured for group C and group D who received BSSO combined with Le Fort I maxillary advancement for the treatment of class III anteroposterior discrepancy were statistically insignificant. $(P>.05)$ The results of Mann Whitney U test for group C and D are shown in Table IV.

\section{Discussion}

PAS narrowing after orthognathic surgery has drawn increasing attention in recent years ${ }^{2}$. The mandible, base of tongue, hyoid bone, velum and pharyngeal walls are intimately related by 
their muscular and ligamentous attachments. The mandible is related to the base of the tongue by the genioglossus muscle ${ }^{22}$. It is likely that the morphology of these structures is influenced and compromised after various orthognathic surgical procedures ${ }^{3}$. Many studies investigated pharyngeal airway changes after various orthognathic surgical procedures. However, the findings of these studies have been somewhat confusing in terms of difference between genders because many of them had used either mixed population of male and female or only female population ${ }^{2,3,6,8,9}$. There is only a few reports comparing the effects of mandibular setback or two-jaws surgery on pharyngeal airway between two different genders ${ }^{13,19}$.

Studies have shown that OSA was more common in males than females despite the fact that females have smaller pharyngeal airway size than males ${ }^{11,12}$. In a study by Mohsenin ${ }^{12}$, males demonstrated to have a greater reductions in pharyngeal airway dimensions with retrusive movement of the mandible, thus, suggesting a greater reduction in pharyngeal airway with mandibular setback surgical procedures in males ${ }^{12}$. It is known that males and females differ with regard to the size and morphology of upper airway structures ${ }^{13,18}$. Moreover, it has been shown that women have greater genioglossal muscle tone than men, suggesting greater defence of the upper airway ${ }^{15}$. Overall, it has been suggested that the upper airway of females is more stable and less constricting than in males ${ }^{13}$.

Beside, there is only a few reports comparing the effects of mandibular setback or two-jaws surgery on pharyngeal airway between two different genders, all of them used lateral cephalometric radiographs ${ }^{13,19}$. It is possible to observe the pharyngeal airway with conventional cephalometric radiography, however, the observation and measurement of the pharyngeal airway is always limited to the lateral viewing angle ${ }^{9}$.

Cephalometric radiography is an indispensable imaging technique for orthodontic treatment planing but can also be used to provide valuable skeletal information for upper airway morphology. However, it provides only 2-dimensional representation of a 3-dimensional 
structure and it is not useful in providing volumetric data of the airway or evaluating important soft tissue structures such as uvulopalatal complex and BoT. Some authors argued that cephalometric measurements are still used extensively in the assessment of pharyngeal airway with the advantages of its wide availability, simplicity, low expense and ease of comparison with extensive normative data and other studies ${ }^{2,14,18}$. Computed tomography carries significant advantages over plain radiographs as it allows better delineation of soft tissue and air, therefore more accurate measurements for upper airway morphology can be made $^{10}$. Skeletal maxillary and mandibular changes may be described by the change in the sagittal dimension only, whereas the soft tissue pharyngeal changes must be considered in all three dimensions ${ }^{7}$. CT scan is a noninvasive technique that permits a detailed 3D assessment of the entire upper airway and has been validated for quantitative measurements of the pharyngeal CSA ${ }^{3}$. Although, some studies comparing airway dimensions on LCRs and 3D CT, reported significant correlation between the PAS measured with LCR and the volume of the pharyngeal airway on CT, LCR provides no information about the lateral structures and CSA of the upper airway ${ }^{20}$. In addition, in frontal cephalometric radiography, there is often an overlap on hard tissue structures such as anterior teeth, mandible and the pharyngeal airway ${ }^{9}$. Many studies have been performed to assess time dependent pharyngeal airway changes after orthognathic surgery. Hochban et $\mathrm{al}^{7}$ reported that no significant changes in pharyngeal dimensions could be seen on cephalometric follow-up at 3 months and 1 year, respectively, as compared to the 1-week postoperative situation ${ }^{7}$. Chen et $\mathrm{al}^{2}$ reported that changes in airway measurements showed significant differences from before surgery to 3-6 months after surgery and 2 years after surgery whereas changes from 3-6 months after surgery to 2 years after surgery showed no significant changes ${ }^{2}$. Kawamata et $\mathrm{al}^{9}$ found that there was a significant pharyngeal narrowing 3 months after surgery and no significant tendency to recover in the average rate of pharyngeal narrowing at either 6 months or 1 year after surgery ${ }^{9}$. Kawakami 
et ${ }^{8}$ al suggested that 1 month after surgery was adequate to let the postoperative swelling in the soft tissue to settle since the narrowed airway was also a result of this ${ }^{8}$. In deference to these results we selected 3 months as the post-surgical time frame.

Few studies revealed that although various variables of pharyngeal airway demonstrated sex dimorphism, oropharyngeal space and minimal pharyngeal airway space did not demonstrate sex dimorphism ${ }^{18,21}$. Samman et al. ${ }^{18}$ suggested that although the majority of airway measurements demonstrate sex dimorphism, those that are most important to the patency of the airway as oropharyngeal space and minimal pharyngeal airway space are not dimorphic ${ }^{18}$. Our results revealed that oropharyngeal airway changes at the level of SP and BoT are not dimorphic, thus, supported the findings of Shen et al. ${ }^{18}$ and Samman et al. ${ }^{21}$.

Nakagawa et al. ${ }^{13}$ found significant difference in changes by gender in linear measurements of the pharyngeal airway led to a difference in changes in the cross sectional area of the pharynx by conventional lateral cephalometric radiographs. They reported that there was a significant decrease in the cross-sectional area of the oropharynx after mandibular setback surgery in both genders; however, for cross-sectional area of the hypopharynx, male patients showed a significant decrease postoperatively, whereas female patients showed no significant changes ${ }^{13}$. Our results was consistent with Nakagawa et al.'s ${ }^{13}$ findings. In both gender who received mandibular setback surgery alone, APD and CSA significantly decreased in either SP or BoT. However, there were no significant difference between males and females.

Samman et al. ${ }^{19}$ declared that some gender differences in the airway changes were evident after various orthognathic surgical procedures in a cephalometric study. They reported that minimal pharyngeal, hypopharyngeal and oropharyngeal spaces were decreased after surgical correction in male Class III subjects whereas no significant change was noted in female Class III subjects ${ }^{19}$. In the same study, Samman et al. ${ }^{19}$ revealed that in male Class III subjects, the most notable change was a decrease in the dimension of minimal pharyngeal and 
hypopharyngeal spaces while there was a decrease in the total pharyngeal area but no change in the dimension of the nasopharyngeal space in female Class III subjects after correction by mandibular setback surgery alone ${ }^{19}$. They also reported that male subjects displayed a decrease in the dimension of minimal pharyngeal airway and oropharyngeal spaces whereas no significant change was noted in female Class III subjects after correction by two-jaws surgery ${ }^{19}$. In our study, we found that most narrowing part of the pharyngeal airway was CSA at the level of SP and BoT for both genders who received mandibular setback surgery alone whereas APD at the level of SP and BoT who received two-jaws surgery. In contrast to Samman et al.'s ${ }^{19}$ findings, our study did not reveal any difference of the oropharyngeal airway change patterns between males and females in neither mandibular setback surgery alone nor two-jaws surgery.

Chen et al. ${ }^{2}$ studied on a female population who received mandibular setback or bimaxillary surgery and found that in mandibular setback surgery alone, AP dimension reduction between uvula-PPW and vallecula-PPW was $32 \%$ wheras in bimaxillary surgery, AP dimension reduction between uvula-PPW was $20 \%$ and between vallecula-PPW was $15 \%^{2}$. In our study, AP reduction of SP-PPW and BoT-PPW was $23 \%$ and $20 \%$, respectively, in mandibular setback surgery while $17 \%$ for either SP-PPW or BoT-PPW in two-jaws surgery for females. We also found that AP reduction of SP-PPW and BoT-PPW was 18\% and 21\%, respectively, in mandibular setback surgery while SP-PPW and BoT-PPW was $13 \%$ and 17\%, respectively in two-jaws surgery for males. Unfortunately, there was not a study evaluating the pharyngeal airway performed on a male population who received mandibular setback or two-jaws surgery therefore we were not able to compare our findings.

Small CSA of the airway is likely to explain the presence of obstructive sleep events ${ }^{3}$. Although, in our study, pharyngeal airway change patterns between males and females did not reveal any difference, CSA reduction rates was different between two genders. In our study, 
we observed that average rate of CSA narrowing was $43 \%$ and $33 \%$ at the level of the SPPPW and BoT-PPW, respectively, for males while it was 31\% and $27 \%$ at the level of the SPPPW and BoT-PPW, respectively, for females in mandibular setback surgery. In two-jaws surgery, the average rate of CSA narrowing was $11 \%$ and $8 \%$ at the level of the SP-PPW and and BoT-PPW, respectively, for males while it was $16 \%$ and $10 \%$ at the level of the SP-PPW and and BoT-PPW, respectively, for females. However, none of the patients in both genders complained about any symptoms of obstructive events.

The normal value for PAS based on a lateral cephalometric study was found to be $11 \pm 2 \mathrm{~mm}^{6}$. Hochban et al. ${ }^{7}$ found that the average preoperative PAS in a prognathic patient was $16.6 \mathrm{~mm}$ between BoT and $\mathrm{PPW}^{7}$. Previous studies have shown that OSA was seen frequently in males than females despite the fact that females have smaller pharyngeal airway size than males ${ }^{11,12}$. In recent study, average preoperative PAS between BoT and PPW was 15.0 \pm 3.1 and 15.2 \pm 3.6 for females and males, respectively. Our results supported the findings of Hochban et al. ${ }^{7}$ CSA at the level of BoT was $1.9 \pm 0.8$ and 2,1 \pm 0.8 for females and males, respectively. The differences between two genders was not statistically significant. ( $p>.05)$ In addition, the average post-operative PAS after mandibular setback surgery decreased to slightly under normal values (11.0 \pm 2.5 for females; $10.8 \pm 3.3$ for males) whereas it was higher than normal values (13.4 \pm 2.9 for females; $12.1 \pm 4.0$ for males) in both genders after two-jaws surgery. Post-operative CSA at the level of BoT was 1.6 \pm .07 and $1.8 \pm 0.7$ for females and males, respectively. Post-operative PAS differences between two genders were not statistically significant after either mandibular setback surgery or two-jaws surgery. ( $p>.05$ ) Unfortunately there were not available studies to compare our findings about pre- and post-operative CSA measurements. 


\section{Conclusion}

This study suggested that oropharyngeal airway measurements which are most important to the patency of the airway does not demonstrate sex dimorphism. Therefore, one should expect same pharyngeal airway reduction change patterns for both genders after mandibular setback or two-jaws surgical procedures. More studies are needed to investigate morphologic changes on pharyngeal airway between females and males. 


\section{References}

1. Busby BR, Bailey LJ, Proffit WR, Phillips C, White RP Jr. Long term stability of surgical Class III treatment: a study of 5-year postsurgical results. Int J Adult Orthod Orthognath Surg 2002; 17: 159-170.

2. Chen F, Terada K, Hua Y, Saito I. Effect of bimaxillary surgery and mandibular setback surgery on pharyngeal airway measurements in patients with Class III skeletal deformities. Am J Dentofacial Orthop 2007; 131: 372-377.

3. Degerliyurt K, Ueki K, Hashiba Y, Marukawa K, Nakagawa K, Yamamoto E. A comparative CT evaluation of pharyngeal airway changes in class III patients receiving bimaxillary surgery or mandibular setback surgery. Oral Surg Oral Med Oral Pathol Oral Radiol Endod 2008; 105: 495-502.

4. Enacar A, Aksoy AU, Sencift Y, Haydar B, Aras K. Changes in hypopharyngeal airway space and in tongue and hyoid bone positions following the surgical correction of mandibular prognathism. Int J Adult Orthod Orthognath Surg 1994; 9 :285-290.

5. Guilleminault C, Riley R, Powell N. Sleep apnea in normal subjects following mandibular osteotomy with retrusion. Chest 1985; 88: 776-778.

6. Guven O, Saracoglu U Changes in pharyngeal airway space and hyoid bone positions after body ostectomies and sagittal split ramus osteotomies. J Craniofac Surg 2005;16: 23-30.

7. Hochban W, Schürmann R, Brandenburg U, Conradt R. Mandibular setback for surgical correction of mandibular hyperplasia - does it provoke sleep-related breathing disorders? Int J Oral Maxillofac Surg 1996; 25: 333-338.

8. Kawakami M, Yamamoto K, Fujımoto M, Ohgi K, Inoue M, Kirita T. Changes in tongue and hyoid positions and posterior airway space following mandibular setback surgery. J Cranio-Maxillofac Surg 2005; 33: 107-110. 
9. Kawamata A, Fujishita M, Ariji Y, Ariji E. Three dimensional computed tomographic evaluation of morphologic airway changes after mandibular setback osteotomy for prognatism. Oral Surg Oral Med Oral Pathol Oral Radiol Endod 2000; 89: 278-287.

10. Li HY, Chen NH, Wang CR, Shu YH, Wang PC. Use of 3-dimensional computed tomography scan to evaluate upper airway patency for patients undergoing sleep-disordered breathing surgery. Otolaryngol Head Neck Surg 2003; 129: 336-342.

11. Martin SE, Mathur R, Marshall I, Douglas NJ. The effect of age, sex, obesity and posture on upper airway size. Eur Respir J. 1997; 10: 2087-2090.

12. Mohsenin V. Effects of gender airway collapsibility and severity of obstructive sleep apnea. Sleep Med 2003; 4: 523-529.

13. Nakagawa F, Ono T, Ishiwata Y, Kuroda T. Morphologic changes in upper airway structure following surgical correction of mandibular prognathism. Int $\mathrm{J}$ Adult Orthod Orthognath Surg. 1998; 13: 209-306

14. Ozbek MM, Miyamoto K, Lowe AA, Fleetham JA. Natural head posture, upper airway morphology and obstructive sleep apnea severity in adults. Eur J Orthod 1998: 20: 133-143.

15. Popovic RM, White DP. Influence of gender on waking genioglossal electromyogram and upper airway resistance. Am J Respir Crit Care Med. 1995; 152: 725-731

16. Reyneke JP. Essentials of orthognathic surgery. Illinois: Quintessence Publishing Co, Inc, 2003; p.164.

17. Riley R, Powell R, Guilleminault C. Obstructive sleep apnea syndrome following surgery for mandibular hyperplasia. J Oral Maxillofac Surg 1987; 45: 450-452.

18. Samman N, Mohammadi H, Xia J. Cephalometric norms for the upper airway in a healthy Hong Kong Chinese population. Hong Kong Med J. 2003; 9: 25-30.

19. Samman N, Tang SS, Xia J. Cephalometric study of the upper airway in surgically corrected class III skeletal deformity. Int J Adult Orthod Orthognath Surg 2002; 17: 180-190. 
20. Schwab RJ. Upper airway imaging Clin Chest Med 1998; 19: 33-54.

21. Shen GF, Samman N, Qui WL, Tang YS, Xia J, Huang YL. Cephalometric studies on the upper airway space in normal Chinese. Int J Oral Maxillofac Surg. 1994; 23: 243-247

22. Tiner BD, Waite PD. Surgical and nonsurgical management of onstructive sleep apnea. In: Miloro M, ed: Peterson's principles of oral and maxillofacial surgery. London: BC Decker: 2004; p1301-1310.

23. Tselnik M, Pogrel MA. Assesment of the pharyngeal airway space after mandibular setback surgery. J Oral Maxillofac Surg 2000; 58: 282-285. 


\section{FIGURE LEGENDS}

Figure 1. M1: The level of most superior anterior point of second cervical spine (C2) parallel to Sella-Nasion line to evaluate the airway between soft palate (SP) and posterior pharyngeal wall (PPW)

M2: The level of most superior anterior point of third cervical spine (C3) parallel to SellaNasion to evaluate the airway between base of the tongue (BoT) and posterior pharyngeal wall (PPW)

Figure 2. Anterioposterior (AP) dimension on the midsagittal plane (black arrows) and maximum lateral (LAT) dimension (white arrows) between lateral pharyngeal walls (LPWs) in an orientation perpendicular to the midsagittal plane. A: SP-PPW level B: BoT-PPW level Figure 3. Cross sectional area (CSA) of the airway (Arrows) A: SP-PPW level B: BoT-PPW level

\section{TABLE LEGENDS}

Table 1. Statistical Analysis and reduction ratios for group A and B. APD: Anteroposterior dimension SP: Soft palate PPW: Posterior pharyngeal wall LD: Lateral dimension LPW: Lateral pharyngeal wall CSA: Cross sectional area BoT: Base of tongue Table 2. Statistical Analysis and reduction ratios for group C and D.

Table 3. The comparison of pharyngeal changes in linear and area measurements by Mann Whitney $\mathrm{U}$ test between group A and group B.

Table 4. The comparison of pharyngeal changes in linear and area measurements by Mann Whitney $\mathrm{U}$ test between group $\mathrm{C}$ and group $\mathrm{D}$ 


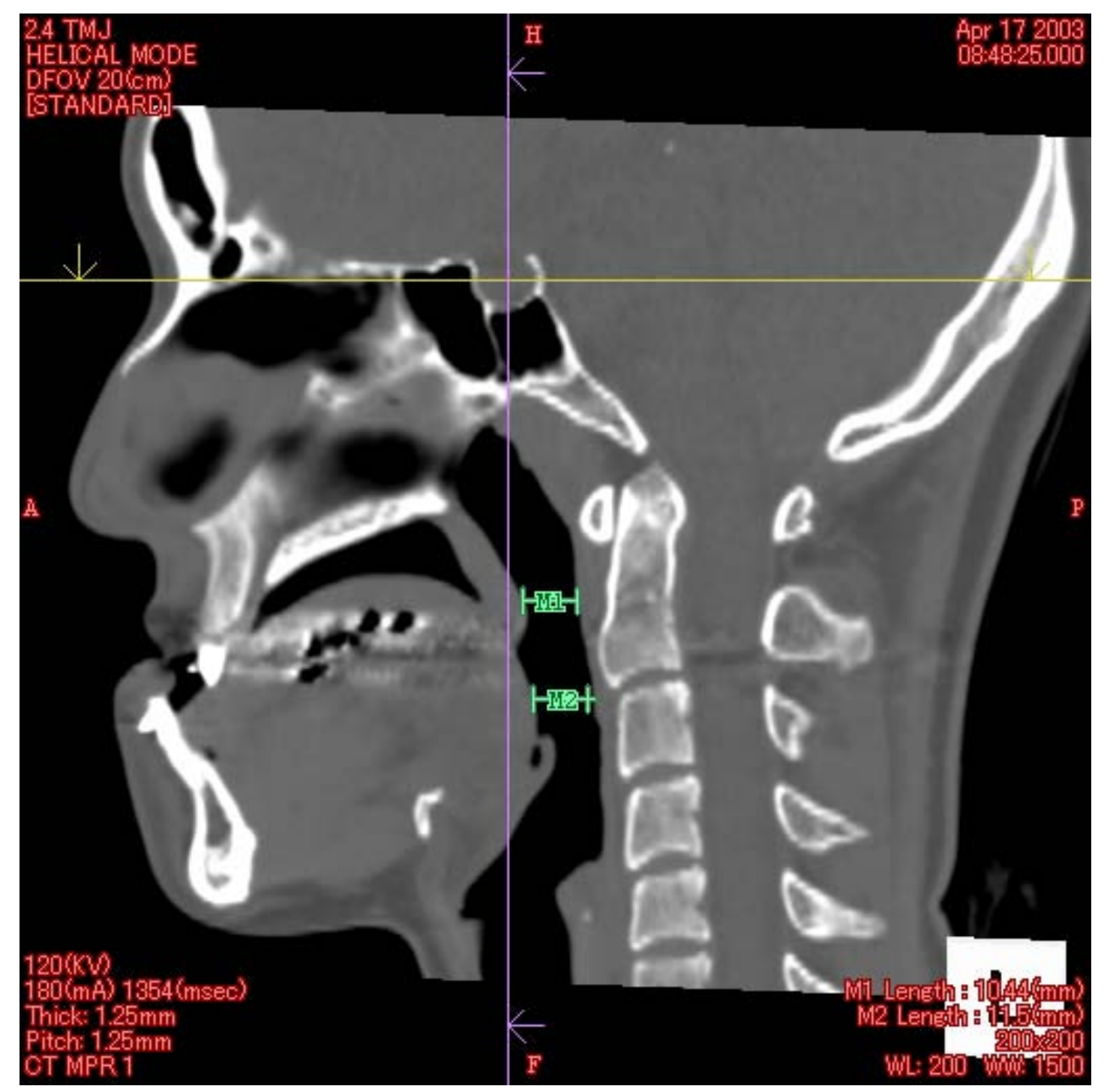

Figure 1. 


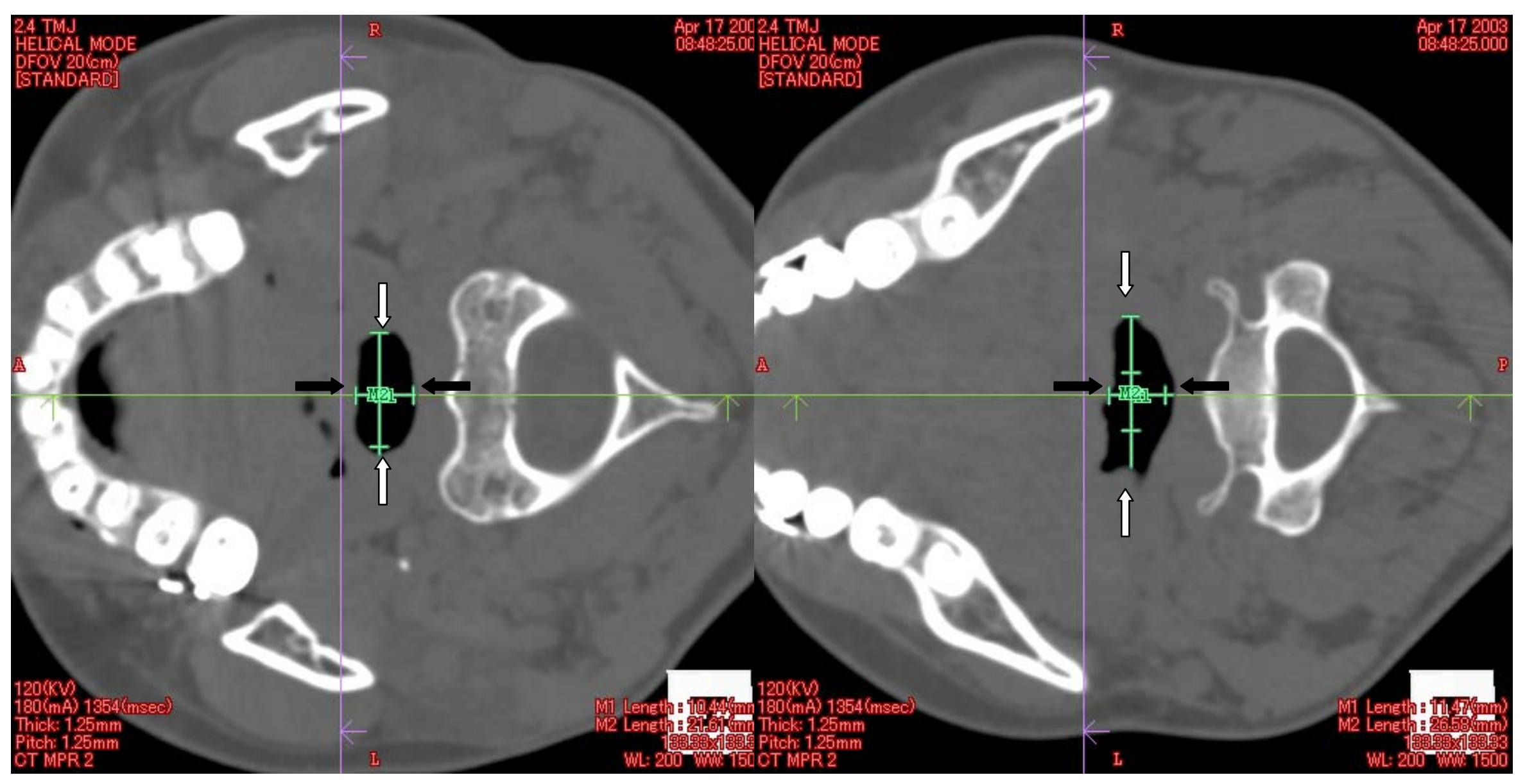

Figure 2. 


\begin{tabular}{|c|c|c|c|c|}
\hline Group A & Pre-Op & Post-Op & $P$ value & Reduction Rate \\
\hline APD SP-PPW & $11.40 \pm 2.86$ & $8.84 \pm 2.11$ & .0004 & $23 \%$ \\
\hline LD LPWs SP-PPW & $20.72 \pm 5.416$ & $18.22 \pm 4.22$ & .0680 & $12 \%$ \\
\hline CSA SP-PPW & $1.27 \pm 0.60$ & $0.87 \pm 0.40$ & .0016 & $31 \%$ \\
\hline APD BoT-PPW & $13.24 \pm 3.14$ & $11.04 \pm 2.45$ & .0003 & $20 \%$ \\
\hline LD LPWs BoT-PPW & $24.80 \pm 4.78$ & $23.15 \pm 5.03$ & .1128 & $6 \%$ \\
\hline CSA BoT-PPW & $1.73 \pm 0.73$ & $1.26 \pm 0.46$ & .0031 & $27 \%$ \\
\hline Group B & Pre-Op & Post-Op & $P$ value & Reduction Rate \\
\hline APD SP-PPW & $10.25 \pm 3.05$ & $8.57 \pm 2.51$ & .0180 & $20 \%$ \\
\hline LD LPWs SP-PPW & $22.08 \pm 5.49$ & $19.30 \pm 4.09$ & .0630 & $13 \%$ \\
\hline CSA SP-PPW & $1.78 \pm 0.93$ & $1.01 \pm 0.25$ & .0180 & $43 \%$ \\
\hline APD BoT-PPW & $13.54 \pm 3.31$ & $10.76 \pm 3.26$ & 0180 & $21 \%$ \\
\hline LD LPWs BoT-PPW & $24.57 \pm 4.12$ & $22.56 \pm 3.74$ & .1763 & $8 \%$ \\
\hline CSA BoT-PPW & $2.13 \pm 0.82$ & $1.44 \pm 0.71$ & .0180 & $33 \%$ \\
\hline
\end{tabular}

Table 1. 


\begin{tabular}{|c|c|c|c|c|}
\hline Group C & Pre-Op & Post-Op & $P$ value & Reduction Rate \\
\hline APD SP-PPW & $13.84 \pm 5.17$ & $11.53 \pm 3.70$ & .0003 & $17 \%$ \\
\hline LD LPWs SP-PPW & $22.64 \pm 5.75$ & $21.14 \pm 6.26$ & .3088 & $7 \%$ \\
\hline CSA SP-PPW & $1.85 \pm 1.01$ & $1.55 \pm 0.88$ & .1488 & $16 \%$ \\
\hline APD BoT-PPW & $16.07 \pm 2.84$ & $13.37 \pm 2.90$ & .0003 & $17 \%$ \\
\hline LD LPWs BoT-PPW & $25.55 \pm 5.17$ & $24.92 \pm 4.93$ & .5540 & $2 \%$ \\
\hline CSA BoT-PPW & $2.11 \pm 0.76$ & $1.89 \pm 0.73$ & .1422 & $10 \%$ \\
\hline Group D & Pre-Op & Post-Op & $P$ value & Reduction Rate \\
\hline APD SP-PPW & $12.02 \pm 5.92$ & $9,75 \pm 4.06$ & .0277 & $18 \%$ \\
\hline LD LPWs SP-PPW & $21.59 \pm 8.48$ & $21.01 \pm 7.51$ & .7532 & $3 \%$ \\
\hline CSA SP-PPW & $1.66 \pm 0.88$ & $1.47 \pm 0.81$ & .5294 & $11 \%$ \\
\hline APD BoT-PPW & $14.58 \pm 4.72$ & $12.11 \pm 4.04$ & .0277 & $17 \%$ \\
\hline LD LPWs BoT-PPW & $26.13 \pm 9.39$ & $25.96 \pm 9.37$ & .6002 & $1 \%$ \\
\hline CSA BoT-PPW & $2.02 \pm 0.99$ & $1.85 \pm 0.77$ & .8389 & $8 \%$ \\
\hline
\end{tabular}

Table 2. 
$\begin{array}{ll}\text { Pre-Op } & \text { Post-Op }\end{array}$

\begin{tabular}{|c|c|c|c|c|c|c|}
\hline & Group A & Group B & $P$ value & Group A & Group B & $P$ value \\
\hline APD SP-PPW & $11.40 \pm 2.86$ & $10.25 \pm 3.05$ & .8489 & $8.84 \pm 2.11$ & $8.57 \pm 2.51$ & .5254 \\
\hline LD LPWs SP-PPW & $20.72 \pm 5.416$ & $22.08 \pm 5.49$ & .0610 & $18.22 \pm 4.22$ & $19.30 \pm 4.09$ & .1274 \\
\hline CSA SP-PPW & $1.27 \pm 0.60$ & $1.78 \pm 0.93$ & .1623 & $0.87 \pm 0.40$ & $1.01 \pm 0.25$ & .1356 \\
\hline APD BoT-PPW & $13.24 \pm 3.14$ & $13.54 \pm 3.31$ & .5463 & $11.04 \pm 2.45$ & $10.76 \pm 3.26$ & .6114 \\
\hline LD LPWs BoT-PPW & $24.80 \pm 4.78$ & $24.57 \pm 4.12$ & .2400 & $23.15 \pm 5.03$ & $22.56 \pm 3.74$ & .4273 \\
\hline CSA BoT-PPW & $1.73 \pm 0.73$ & $2.13 \pm 0.82$ & .1197 & $1.26 \pm 0.46$ & $1.44 \pm 0.71$ & .4652 \\
\hline
\end{tabular}

Table 3. 


\section{Pre-Op}

\section{Group C}

APD SP-PPW

LD LPWs SP-PPW

CSA SP-PPW

APD BoT-PPW

LD LPWs BoT-PPW

CSA BoT-PPW
$13.84 \pm 5.17$

$22.64 \pm 5.75$

$1.85 \pm 1.01$

$16.07 \pm 2.84$

$25.55 \pm 5.17$

$2.11 \pm 0.76$

\section{Group D}

$12.02 \pm 5.92$

.2076

$11.53 \pm 3.70$

$9,75 \pm 4.06$

.2936

$1.59+8.48$

.8336

$21.14 \pm 6.26$

$21.01 \pm 7.51$

.8886

$1.66 \pm 0.88$

.7794

$1.55 \pm 0.88$

$1.47 \pm 0.81$

.7624

.4838

$13.37 \pm 2.90$

$12.11 \pm 4.04$

.6744

$26.13 \pm 9.39$

.3270

$24.92 \pm 4.93$

.9442

$1.89 \pm 0.73$

$1.85 \pm 0.77$

.7794

Table 4. 